\title{
Asymmetric structures, discontinuous contractions and iterative approximation of fixed and periodic points
}

\author{
Kazimierz Włodarczyk ${ }^{*}$ and Robert Plebaniak
}

\section{"Correspondence:}

wlkzxa@math.uni.lodz.pl

Department of Nonlinear Analysis,

Faculty of Mathematics and

Computer Science, University of Łódź, Banacha 22, Łódź, 90-238,

Poland

\begin{abstract}
In quasi-pseudometric spaces $(X, p)$ (not necessarily Hausdorff), the concepts of the left quasi-closed maps (generalizing continuous maps) and generalized quasi-pseudodistances $J: X \times X \rightarrow[0, \infty)$ (generalizing in metric spaces: metrics, Tataru distances, $w$-distances of Kada et al., $\tau$-distances of Suzuki and $\tau$-functions of Lin and $\mathrm{Du}$ ) are introduced, the asymmetric structures on $X$ determined by $J$ (generalizing the asymmetric structure on $X$ determined by quasi-pseudometric $p$ ) are described and the contractions $T: X \rightarrow X$ with respect to $J$ (generalizing Banach and Rus contractions) are defined. Moreover, if $(X, p)$ are left sequentially complete (in the sense of Reilly, Subrahmanyam and Vamanamurthy), then, for these contractions $T: X \rightarrow X$ such that $T^{[q]}$ is left quasi-closed for some $q \in \mathbb{N}$, the global minimum of the map $x \rightarrow J\left(x, T^{[q]}(x)\right)$ is studied and theorems concerning the existence of global optimal approximate solutions of the equation $T^{[q]}(x)=x$ are established. The results are new in quasi-pseudometric and quasi-metric spaces and even in metric spaces. Examples showing the difference between our results and the well-known ones are provided. In the literature the fixed and periodic points in not Hausdorff spaces were not studied.
\end{abstract}

MSC: 41A65; 47H09; 47H10; 37C25; 54E15

Keywords: global optimal approximate solution; contraction; left quasi-closed map; periodic point; fixed point; quasi-pseudometric space; generalized quasi-pseudodistance

\section{Introduction}

Let $X$ be a space and let $T: X \rightarrow X$. By Fix $(T)$ and $\operatorname{Per}(T)$ we denote the sets of all fixed points and periodic points of $T: X \rightarrow X$, respectively, i.e., $\operatorname{Fix}(T)=\{w \in X: w=T(w)\}$ and $\operatorname{Per}(T)=\left\{w \in X: w=T^{[q]}(w)\right.$ for some $\left.q \in \mathbb{N}\right\}$.

Motivated by results from the large literature concerning the global optimal approximate solution theorems, in which the spaces $X$ are metric, the maps $T: X \rightarrow X$ are contractions of Banach or Rus types and optimal approximate solutions belong to the set $\operatorname{Fix}(T)$, our main interest of this paper is the following.

Question 1.1 Let $(X, p)$ be a quasi-pseudometric. Are there generalized pseudodistance $J: X \times X \rightarrow[0, \infty)$ generalizing $p$ (generalizing asymmetric structure on $X$ determined by a quasi-pseudometric $p$ ), the map $T: X \rightarrow X$ satisfying the condition $\exists_{\lambda \in[0,1)} \forall_{x \in X}\left\{J\left(T(x), T^{[2]}(x)\right) \leq \lambda J(x, T(x))\right\}$ and $q \in \mathbb{N} \backslash\{1\}$ such that the map $x \rightarrow J\left(x, T^{[q]}(x)\right)$ 
attains its global minimum at the approximate solution $w$ of the equation $T^{[q]}(x)=x$ and $w$ satisfies the equation $J\left(w, T^{[q]}(w)\right)=0$ but: (i) the space $(X, p)$ is not Hausdorff; (ii) $T$ does not satisfy the condition $\exists_{\lambda \in[0,1)} \forall_{x \in X}\left\{p\left(T(x), T^{[2]}(x)\right) \leq \lambda p(x, T(x))\right\}$; (iii) $T$ is not continuous in $(X, p)$; (iv) $\operatorname{Fix}(T)=\varnothing$ ?

Our aim in this paper is to answer the question affirmatively.

The fixed point theory is currently a very active field. In this theory, the notion of contractivity introduced by Banach belongs to the most fundamental mathematical ideas and the following theorem concerning the existence of global optimal approximate solution has important generalizations and applications.

Theorem 1.1 (Banach [1], Caccioppoli [2]) Let $(X, d)$ be a complete metric space. If $T$ : $X \rightarrow X$ satisfies the contractive condition

$$
\exists_{\lambda \in[0,1)} \forall_{x, y \in X}\{d(T(x), T(y)) \leq \lambda d(x, y)\},
$$

then $T$ has a unique fixed point $w$ in $X$ and $\forall_{w^{0} \in X}\left\{\lim _{m \rightarrow \infty} T^{[m]}\left(w^{0}\right)=w\right\}$.

This shows that the map $x \rightarrow d(x, T(x))$ is continuous and this map attains its global minimum at the approximate unique solution of the equation $x=T(x)$; we note that here $d$ and $T$ are continuous, the space $(X, d)$ is Hausdorff and $d$ determines the symmetric structure on $X$.

It is important to observe that the map $T: X \rightarrow X$ satisfying (1.1) satisfies (Rus [3])

$$
\exists_{\lambda \in[0,1)} \forall_{x \in X}\left\{d\left(T(x), T^{[2]}(x)\right) \leq \lambda d(x, T(x))\right\}
$$

and the converse is not true. In general, the maps $T: X \rightarrow X$ satisfying (1.2) are not continuous.

Banach's global optimal approximate solution theorem has inspired a large body of work over the last 40 years.

Rus [3] proved that the conclusions of Theorem 1.1 hold without the uniqueness assertion but under a slightly weaker contractive assumption; for other results in this direction, we refer to Subrahmanyam [4], Kasahara [5] and Hicks and Rhoades [6].

Theorem 1.2 (Rus [3]) Let $(X, d)$ be a complete metric space and let $T: X \rightarrow X$. If $T$ is a continuous map satisfying contractive condition (1.2), then $\operatorname{Fix}(T) \neq \varnothing$ and $\forall_{w^{0} \in X} \exists_{w \in \operatorname{Fix}(T)}\left\{\lim _{m \rightarrow \infty} T^{[m]}\left(w^{0}\right)=w\right\}$.

The concepts of the asymmetric structures became established and investigated in mathematics and its applications. For details, see, e.g., [7] in normed spaces; [8-11] in metric spaces; [12] in uniform spaces; and [13-22] in quasi-gauge, cone uniform and uniform spaces.

There are many different generalizations of Theorems 1.1 and 1.2 in the literature where the distances are more general than $d$. In particular, we give some references where various contractions of Rus type are naturally defined. We refer to the works [8] and [10, 23-26] where in complete metric spaces $(X, d)$ the distance $d$ in condition (1.2) is replaced by $w$ distances and $\tau$-distances, respectively. The authors [13] introduced and examined, in not 
necessarily sequentially complete uniform spaces, contractions of Rus type with respect to the families of generalized pseudodistances.

In this paper, in quasi-pseudometric spaces $(X, p)$ (not necessarily Hausdorff), the concepts of the left quasi-closed maps (generalizing continuous maps) and generalized quasipseudodistances $J: X \times X \rightarrow[0, \infty)$ (generalizing the quasi-pseudometrics $p$ in quasipseudometric spaces $(X, p)$, and generalizing in metric spaces $(X, d)$ : metrics $d$, distances of Tataru [11], $w$-distances of Kada et al. [8], $\tau$-distances of Suzuki [10] and $\tau$-functions of Lin and $\mathrm{Du}$ [9]) are introduced. Next, the asymmetric structures on $X$ determined by $J$ (generalizing asymmetric structure on $X$ determined by quasi-pseudometrics $p$ ) are described and the contractions $T: X \rightarrow X$ with respect to $J$ (generalizing Banach and Rus contractions) are defined. Moreover, if $(X, p)$ are left sequentially complete (in the sense of Reilly et al. [27]), then, for these contractions $T: X \rightarrow X$, assuming that $T^{[q]}$ is left quasiclosed for some $q \in \mathbb{N}$, the global minimum of the map $x \rightarrow J\left(x, T^{[q]}(x)\right)$ is studied and theorems concerning the existence of global optimal approximate solutions of the equation $T^{[q]}(x)=x$ are established.

In addition, the provided examples illustrating generalized quasi-pseudodistances and our theorems, describe the techniques which enable one to compute the periodic and fixed points as well as to give precise information about the difference between our results and the well-known ones. The techniques require considerably more machinery from fixed point theory, asymmetric structures and iterative approximation.

Note that quasi-pseudometric spaces generalize quasi-metric and metric spaces and the studies of asymmetric structures in quasi-pseudometric and quasi-metric spaces and their applications to problems in theoretical computer science are important.

\section{Definitions, notations and statement of results}

Definition 2.1 [28-31] Let $X$ be a nonempty set.

(i) A map $p: X \times X \rightarrow[0, \infty)$ is called quasi-pseudometric on $X$ if it satisfies the two conditions:

$$
\forall_{x \in X}\{p(x, x)=0\}
$$

and

$$
\forall_{x, y, z \in X}\{p(x, z) \leq p(x, y)+p(y, z)\}
$$

For given quasi-pseudometric $p$ on $X$, a pair $(X, p)$ is called quasi-pseudometric space.

(ii) A quasi-pseudometric space $(X, p)$ is called Hausdorff if

$$
\forall_{x, y \in X}\{x \neq y \Rightarrow p(x, y)>0 \vee p(y, x)>0\}
$$

(iii) A map $p: X \times X \rightarrow[0, \infty)$ is called quasi-metric on $X$ if it satisfies (2.1), (2.2) and

$$
\forall_{x, y \in X}\{p(x, y)=0 \Rightarrow x=y\}
$$

For given quasi-metric $p$ on $X$, a pair $(X, p)$ is called quasi-metric space. 
(iv) A map $p: X \times X \rightarrow[0, \infty)$ is called metric on $X$ if it satisfies (2.1)-(2.3) and $\forall_{x, y \in X}\{p(x, y)=p(y, x)\}$. For given metric $p$ on $X$, a pair $(X, p)$ is called metric space.

In order to investigate the new contractivity in quasi-pseudometric spaces, we need to introduce the concept of 'generalized quasi-pseudodistances'.

Definition 2.2 Let $(X, p)$ be a quasi-pseudometric space. The map $J: X \times X \rightarrow[0, \infty)$ is said to be a generalized quasi-pseudodistance on $X$ if the following two conditions hold:

(J1) $\forall_{x, y, z \in X}\{J(x, z) \leq J(x, y)+J(y, z)\}$; and

(J2) For any sequences $\left(x_{m}: m \in \mathbb{N}\right)$ and $\left(y_{m}: m \in \mathbb{N}\right)$ in $X$ satisfying

$$
\forall_{\varepsilon>0} \exists_{k \in \mathbb{N}} \forall_{n, m \in \mathbb{N} ; k \leq m<n}\left\{J\left(x_{m}, x_{n}\right)<\varepsilon\right\}
$$

and

$$
\forall_{\varepsilon>0} \exists_{k \in \mathbb{N}} \forall_{m \in \mathbb{N} ; k \leq m}\left\{J\left(x_{m}, y_{m}\right)<\varepsilon\right\},
$$

the following holds:

$$
\forall_{\varepsilon>0} \exists_{k \in \mathbb{N}} \forall_{m \in \mathbb{N} ; k \leq m}\left\{p\left(x_{m}, y_{m}\right)<\varepsilon\right\} .
$$

Remark 2.1 Let $(X, p)$ be a quasi-pseudometric space and let $\mathbb{J}_{(X, p)}$ be a class defined as follows: $\mathbb{J}_{(X, p)}=\{J: J$ is generalized quasi-pseudodistance on $X\}$. Then:

(a) $\mathbb{J}_{(X, p)} \neq \varnothing$ since $p \in \mathbb{J}_{(X, p)}$;

(b) $\mathbb{J}_{(X, p)} \neq\{p\}$, see Examples 4.2 and 4.7;

(c) Each quasi-pseudometric is a generalized quasi-pseudodistance, but converse is not true (see Section 4).

One can prove the following proposition.

Proposition 2.1 Let $(X, p)$ be a Hausdorff quasi-pseudometric space and let $J \in \mathbb{J}_{(X, p)}$. Then

$$
\forall_{x, y \in X}\{x \neq y \Rightarrow J(x, y)>0 \vee J(y, x)>0\} .
$$

Proof Assume that there are $x \neq y, x, y \in X$, such that $J(x, y)=J(y, x)=0$. Then $J(x, x)=0$ since, by using (J1), it follows that $J(x, x) \leq J(x, y)+J(y, x)=0$. Defining the sequences $\left(x_{m}\right.$ : $m \in \mathbb{N})$ and $\left(y_{m}: m \in \mathbb{N}\right)$ in $X$ by $x_{m}=x$ and $y_{m}=y$ or $x_{m}=y$ and $y_{m}=x$ for $m \in \mathbb{N}$, and observing that $J(x, y)=J(y, x)=J(x, x)=0$, we see that (2.4) and (2.5) for these sequences hold. Then, by (J2), (2.6) holds, so it is $p(x, y)=p(y, x)=0$. But this is a contradiction since $(X, p)$ is Hausdorff and thus $x \neq y$ implies $p(x, y)>0$ or $p(y, x)>0$.

Recall the following definition.

Definition $2.3[27,28,32]$ Let $(X, p)$ be a quasi-pseudometric space.

(i) We say that a sequence $\left(w_{m}: m \in \mathbb{N}\right)$ is left Cauchy sequence in $X$ if

$$
\forall_{\varepsilon>0} \exists_{k \in \mathbb{N}} \forall_{m, n \in \mathbb{N} ; k \leq m<n}\left\{p\left(w_{m}, w_{n}\right)<\varepsilon\right\} .
$$


(ii) We say that a sequence $\left(w_{m}: m \in \mathbb{N}\right)$ is left convergent in $X$ if

$$
\exists_{w \in X} \forall_{\varepsilon>0} \exists_{k \in \mathbb{N}} \forall_{m \in \mathbb{N} ; k \leq m}\left\{p\left(w, w_{m}\right)<\varepsilon\right\}
$$

(which we write as $\lim _{m \rightarrow \infty}^{L} w_{m}=w$ ).

(iii) If every left Cauchy sequence in $X$ is left convergent to some point in $X$, then $(X, p)$ is called left sequentially complete quasi-pseudometric space.

Using this we can define the following natural generalization of continuity.

Definition 2.4 Let $(X, p)$ be a left sequentially complete quasi-pseudometric space, let $T$ : $X \rightarrow X$ and let $q \in \mathbb{N}$. The map $T^{[q]}$ is called left quasi-closed in $X$ if every sequence $\left(w_{m}\right.$ : $m \in \mathbb{N})$ in $T^{[q]}(X)$, left converging to each point of the set $W \subset X$ and having subsequences $\left(v_{m}: m \in \mathbb{N}\right)$ and $\left(u_{m}: m \in \mathbb{N}\right)$ satisfying $\forall_{m \in \mathbb{N}}\left\{v_{m}=T^{[q]}\left(u_{m}\right)\right\}$, has the property $\exists_{w \in W}\{w=$ $\left.T^{[q]}(w)\right\}$.

By $\operatorname{Fix}(T)$ and $\operatorname{Per}(T)$ we denote the sets of all fixed points and periodic points of $T: X \rightarrow X$, respectively, i.e., $\operatorname{Fix}(T)=\{w \in X: w=T(w)\}$ and $\operatorname{Per}(T)=\{w \in X: w=$ $T^{[q]}(w)$ for some $\left.q \in \mathbb{N}\right\}$.

Motivated by papers $[8,10,23-26]$, we raise a question.

Question 2.1 In quasi-pseudometric spaces (and thus also in quasi-metric and in metric spaces), is it possible to find an effective construction of a condition of Rus type with respect to generalized quasi-pseudodistances and techniques for obtaining periodic and fixed point theorems for left quasi-closed maps satisfying this condition?

The purpose of this paper is to answer this question in the affirmative. The first result in this direction is the following.

Theorem 2.1 Assume that $(X, p)$ is a left sequentially complete quasi-pseudometric space, the map $J: X \times X \rightarrow[0, \infty)$ is a generalized quasi-pseudodistance on $X$ and the map $T$ : $X \rightarrow X$ satisfies

(S1) $\exists_{\lambda \in[0,1)} \forall_{x \in X}\left\{J\left(T(x), T^{[2]}(x)\right) \leq \lambda J(x, T(x))\right\}$.

The following statements hold.

(A) For each $w^{0} \in X$ there exists a nonempty set $M\left(w^{0}\right) \subset X$ such that the sequence $\left(T^{[m]}\left(w^{0}\right): m \in \mathbb{N}\right)$ is left convergent to each point $w \in M\left(w^{0}\right)$; i.e., (A1) For each $w^{0} \in X, M\left(w^{0}\right)=\left\{w \in X: \lim _{m \rightarrow \infty}^{L} T^{[m]}\left(w^{0}\right)=w\right\} \neq \varnothing$.

(B) If: $\left(\mathrm{b}_{1}\right) T^{[q]}$ is left quasi-closed in $X$ for some $q \in \mathbb{N}$, then: (B1) $\operatorname{Fix}\left(T^{[q]}\right) \neq \varnothing$; (B2) For each $w^{0} \in X$ there exists $w \in \operatorname{Fix}\left(T^{[q]}\right)$ such that $w \in M\left(w^{0}\right)$ (i.e., $\left.\lim _{m \rightarrow \infty}^{L} T^{[m]}\left(w^{0}\right)=w\right)$; and (B3) For each $w \in \operatorname{Fix}\left(T^{[q]}\right), J(w, T(w))=J(T(w), w)=0$.

(C) If: $\left(\mathrm{c}_{1}\right)(X, p)$ is a Hausdorff space; and $\left(\mathrm{c}_{2}\right) w \in \operatorname{Fix}\left(T^{[q]}\right)$ for some $q \in \mathbb{N}$, then: $(\mathrm{C} 1)$ $w \in \operatorname{Fix}(T) ;$ and $(\mathrm{C} 2) J(w, w)=0$.

A version of Definition 2.2 in metric spaces is as follows.

Definition 2.5 Let $(X, p)$ be a metric space. The map $J: X \times X \rightarrow[0, \infty)$, is said to be a generalized pseudodistance on $X$ if the following two conditions hold: (J1) 
$\forall_{x, y, z \in X}\{J(x, z) \leq J(x, y)+J(y, z)\}$; and (J2) For any sequences $\left(x_{m}: m \in \mathbb{N}\right)$ and $\left(y_{m}: m \in \mathbb{N}\right)$ in $X$ satisfying $\lim _{n \rightarrow \infty} \sup _{m>n} J\left(x_{n}, x_{m}\right)=0$ and $\lim _{m \rightarrow \infty} J\left(x_{m}, y_{m}\right)=0$, the following holds $\lim _{m \rightarrow \infty} p\left(x_{m}, y_{m}\right)=0$.

The following is a metric analog of Theorem 2.1.

Theorem 2.2 Assume that $(X, p)$ is a complete metric space, the map $J: X \times X \rightarrow[0, \infty)$ is a generalized pseudodistance on $X$ and the map $T: X \rightarrow X$ satisfies

(S2) $\exists_{\lambda \in[0,1)} \forall_{x \in X}\left\{J\left(T(x), T^{[2]}(x)\right) \leq \lambda J(x, T(x))\right\}$.

The following statements hold:

(A) For any $w^{0} \in X$ there exists $w \in X$ such that $\lim _{m \rightarrow \infty} T^{[m]}\left(w^{0}\right)=w$.

(B) If: $\left(\mathrm{b}_{1}\right) T^{[q]}$ is continuous in $X$ for some $q \in \mathbb{N}$, then, for any $w^{0} \in X$, there exists $w \in X$ such that: (B1) $\lim _{m \rightarrow \infty} T^{[m]}\left(w^{0}\right)=w$; (B2) $w \in \operatorname{Fix}(T)$; and (B3) $J(w, w)=0$.

Remark 2.2 If we assume that $J=p$ and $q=1$, then a special case of Theorem 2.2 gives Theorem 1.2.

\section{Proof of Theorem 2.1}

Proof In the sequel, for each $w^{0} \in X$, a sequence $\left(w^{m}: m \in\{0\} \cup \mathbb{N}\right)$ is defined by $w^{m}=$ $T^{[m]}\left(w^{0}\right)$ for $m \in\{0\} \cup \mathbb{N}$; we see that $\forall_{m \in \mathbb{N}}\left\{w^{m}=T\left(w^{m-1}\right)\right\}$ and $T^{[0]}=I_{X}$.

(A) The proof will be divided into four steps.

Step 1. We show that

$$
\forall_{w^{0} \in X}\left\{\lim _{m \rightarrow \infty} \sup \left\{J\left(w^{m}, w^{n}\right): n>m\right\}=0\right\}
$$

Indeed, if $w^{0} \in X$ is arbitrary and fixed, $m, n \in \mathbb{N}$ and $n>m$, then, by (J1) and (S1),

$$
\begin{aligned}
\lim _{m \rightarrow \infty} \sup \left\{J\left(w^{m}, w^{n}\right): n>m\right\} & \leq \lim _{m \rightarrow \infty} \sup \left\{\sum_{i=m}^{n-1} J\left(w^{i}, w^{i+1}\right): n>m\right\} \\
& \leq \lim _{m \rightarrow \infty} \sup \left\{\sum_{i=m}^{n-1} \lambda^{i} J\left(w^{0}, w^{1}\right): n>m\right\} \\
& \leq \lim _{m \rightarrow \infty} \lambda^{m} J\left(w^{0}, w^{1}\right) /(1-\lambda)=0 .
\end{aligned}
$$

Step 2. We show that

$$
\forall_{w^{0} \in X} \forall_{\varepsilon>0} \exists_{k \in \mathbb{N}} \forall_{m \in \mathbb{N} ; k \leq m} \forall_{n \in \mathbb{N} ; m<n}\left\{J\left(w^{m}, w^{n}\right)<\varepsilon\right\} .
$$

Indeed, by (3.1), we get $\forall_{w^{0} \in X} \forall_{\varepsilon>0} \exists_{k \in \mathbb{N}} \forall_{m \in \mathbb{N} ; k \leq m}\left\{\sup \left\{J\left(w^{m}, w^{n}\right): n>m\right\}<\varepsilon\right\}$. This implies (3.2).

Step 3. For each $w^{0} \in X$ the sequence $\left(w^{m}: m \in N\right)$ is a left Cauchy sequence on $X$. Indeed, let $w^{0} \in X$ be arbitrary and fixed. Then, by (3.2), we have

$$
\forall_{\varepsilon>0} \exists_{k \in \mathbb{N}} \forall_{m \in \mathbb{N} ; k \leq m} \forall_{l \in \mathbb{N}}\left\{J\left(w^{m}, w^{l+m}\right)<\varepsilon\right\} .
$$


Hence, if $l_{0} \in \mathbb{N}$ is arbitrary and fixed and if we define a sequence $\left(v_{m}: m \in \mathbb{N}\right)$ as $v_{m}=w^{l_{0}+m}$ for $m \in \mathbb{N}$, then we get

$$
\forall_{\varepsilon>0} \exists_{k \in \mathbb{N}} \forall_{m \in \mathbb{N} ; k \leq m}\left\{J\left(w^{m}, v_{m}\right)<\varepsilon\right\} .
$$

Now, from (3.2), (3.3) and (J2) of Definition 2.2, we conclude that

$$
\forall_{\varepsilon>0} \exists_{k \in \mathbb{N}} \forall_{m \in \mathbb{N} ; k \leq m}\left\{p\left(w^{m}, v_{m}\right)<\varepsilon\right\} .
$$

The consequence of (3.4) and definition of $\left(v_{m}: m \in \mathbb{N}\right)$ is

$$
\forall_{\varepsilon>0} \exists_{k \in \mathbb{N}} \forall_{m \in \mathbb{N} ; k \leq m}\left\{p\left(w^{m}, w^{l_{0}+m}\right)<\varepsilon\right\}
$$

here $l_{0} \in \mathbb{N}$ is arbitrary and fixed. This gives

$$
\forall_{\varepsilon>0} \exists_{k \in \mathbb{N}} \forall_{m \in \mathbb{N} ; k \leq m} \forall_{l \in \mathbb{N}}\left\{p\left(w^{m}, w^{l+m}\right)<\varepsilon\right\}
$$

Now, let $\varepsilon_{0}>0$ be arbitrary and fixed. From (3.5) we get that

$$
\exists_{k_{0} \in \mathbb{N}} \forall_{m \in \mathbb{N} ; k_{0} \leq m} \forall_{l \in \mathbb{N}}\left\{p\left(w^{m}, w^{l+m}\right)<\varepsilon_{0}\right\} .
$$

We see that if $m$ and $n$ satisfy $k_{0} \leq m<n$, then $n=l_{0}+m$ for some $l_{0} \in \mathbb{N}$. Therefore by (3.6),

$$
p\left(w^{m}, w^{n}\right)=p\left(w^{m}, w^{l_{0}+m}\right)<\varepsilon_{0}
$$

so it is

$$
\exists_{k_{0} \in \mathbb{N}} \forall_{m, n \in \mathbb{N} ; k_{0} \leq m<n}\left\{p\left(w^{m}, w^{n}\right)<\varepsilon_{0}\right\} .
$$

Thus the sequence $\left(w^{m}: m \in \mathbb{N}\right)$ is left Cauchy sequence on $X$.

Step 4. For each $w^{0} \in X$ there exists a nonempty set $M\left(w^{0}\right) \subset X$ such that the sequence $\left(w^{m}: m \in \mathbb{N}\right)$ is left convergent to each point $w \in M\left(w^{0}\right)$.

Indeed, let $w^{0} \in X$ be arbitrary and fixed. By Step 3, the sequence $\left(w^{m}: m \in\{0\} \cup \mathbb{N}\right)$ is left Cauchy on $X$. Hence, since $(X, p)$ is left sequentially complete quasi-pseudometric space, there exists a nonempty subset $M\left(w^{0}\right)$ of $X$, such that the sequence $\left(w^{m}: m \in \mathbb{N}\right)$ is left convergent to each point $w \in M\left(w^{0}\right)$.

(B) We have

$$
w^{m q+k}=T^{[q]}\left(w^{(m-1) q+k}\right) \quad \text { for } k=1,2, \ldots, q \text { and } m \in \mathbb{N} .
$$

Clearly, by Step 4 , for each $k=1,2, \ldots, q$, the sequences $\left(v_{m}=w^{m q+k}: m \in\{0\} \cup \mathbb{N}\right)$ and $\left(u_{m}=w^{(m-1) q+k}: m \in \mathbb{N}\right)$, as subsequences of $\left(w_{m}=w^{m+q}: m \in\{0\} \cup \mathbb{N}\right) \subset T^{[q]}(X)$, are also left convergent to each point of $M\left(w^{0}\right)$; more precisely, $\left(v_{m}: m \in\{0\} \cup \mathbb{N}\right)$ is left convergent to each point of $V=\left\{v \in X: \lim _{m \rightarrow \infty}^{L} v_{m}=v\right\},\left(u_{m}: m \in \mathbb{N}\right)$ is left convergent to each point of $U=\left\{u \in X: \lim _{m \rightarrow \infty}^{L} u_{m}=u\right\}, M\left(w^{0}\right) \subset V$ and $M\left(w^{0}\right) \subset U$. Additionally, 
$\forall_{m \in \mathbb{N}}\left\{v_{m}=T^{[q]}\left(u_{m}\right)\right\}$. Since $T^{[q]}$ is left quasi-closed, by (3.7) and Definition 2.4, we obtain that $\exists_{w \in M\left(w^{0}\right)}\left\{w=T^{[q]}(w)\right\}$.

We show that (B3) holds. Indeed, assume that $w \in \operatorname{Fix}\left(T^{[q]}\right)$ is arbitrary and fixed.

We see that

$$
J(w, T(w))=0 .
$$

Otherwise, $J(w, T(w))>0$. Hence, by (J1) and (S1), since $w=T^{[q]}(w)=T^{[2 q]}(w)$, we get $0<$ $J(w, T(w))=J\left(T^{[2 q]}(w), T^{[2 q]}(T(w))\right)=J\left(T\left(T^{[2 q-1]}(w)\right), T^{[2]}\left(T^{[2 q-1]}(w)\right)\right) \leq \lambda J\left(T\left(T^{[2 q-2]}(w)\right)\right.$, $\left.T^{[2]}\left(T^{[2 q-2]}(w)\right)\right) \leq \lambda^{2} J\left(T\left(T^{[2 q-3]}(w)\right), T^{[2]}\left(T^{[2 q-3]}(w)\right)\right) \leq \cdots \leq \lambda^{2 q} J(w, T(w))<J(w, T(w))$ which is impossible. Therefore, (3.8) holds.

Next, we see that

$$
J(T(w), w)=0 .
$$

Otherwise, $J(T(w), w)>0$. Hence, by (J1), (S1) and (3.8), since $w=T^{[q]}(w)=T^{[2 q]}(w)$ and $q+1<2 q$, we get $0<J(T(w), w)=J\left(T\left(T^{[q]}(w)\right), T^{[2 q]}(w)\right)=J\left(T^{[q+1]}(w), T^{[2 q]}(w)\right) \leq$ $\sum_{i=q+1}^{2 q-1} \lambda^{i} J(w, T(w))=0$ which is impossible. Therefore, (3.9) holds.

(C) From (3.8), (3.9) and the fact that $(X, p)$ is Hausdorff, using Proposition 2.1, we get $T(w)=w$, i.e., $w \in \operatorname{Fix}(T)$.

Finally, by (J1), (3.8) and (3.9), we get $J(w, w) \leq J(w, T(w))+J(T(w), w)=0$.

\section{Examples and comparisons}

In this section we present some examples illustrating the concepts introduced so far.

The following two examples illustrate the concept of a quasi-pseudometric space and generalized quasi-pseudodistances, respectively.

Example 4.1 Let $X \subset \mathbb{R}$ be a nonempty set and let $p: X \times X \rightarrow[0, \infty)$ be defined by the formula

$$
p(x, y)=\left\{\begin{array}{ll}
0 & \text { if } x \geq y, \\
1 & \text { if } x<y
\end{array} \quad x, y \in X\right.
$$

(I.1) The map $p$ is quasi-pseudometric on $X$, and $(X, p)$ is quasi-pseudometric space (see Reilly et al. [27]).

(I.2) $(X, p)$ is Hausdorff. Indeed, let $x \neq y, x, y \in X$. Then, by (4.1), $y>x$ implies $p(x, y)=$ $1>0$ and $x>y$ implies $p(y, x)=1>0$. By Definition 2.1(ii), $(X, p)$ is Hausdorff.

Example 4.2 Let $(X, p)$ be a quasi-pseudometric space. Let the set $E \subset X$, containing at least two different points, be arbitrary and fixed and let $c>0$ satisfy $\delta(E)<c$, where $\delta(E)=$ $\sup \{p(x, y): x, y \in E\}$. Let $J: X \times X \rightarrow[0, \infty)$ be defined by the formula

$$
J(x, y)=\left\{\begin{array}{ll}
p(x, y) & \text { if } E \cap\{x, y\}=\{x, y\}, \\
c & \text { if } E \cap\{x, y\} \neq\{x, y\},
\end{array} \quad x, y \in X .\right.
$$

(II.1) The map J is a generalized quasi-pseudodistance on $X$ (see [14]).

Now, we present the examples illustrating Theorems 2.1 and 2.2. 
Example 4.3 Let $(X, p)$ be a Hausdorff quasi-pseudometric space, where $X=[0,6]$ and let $p: X \times X \rightarrow[0, \infty)$ be as in Example 4.1. Let $E=[0,1) \cup(2,3] \cup\{6\}$ and let

$$
J(x, y)= \begin{cases}p(x, y) & \text { if }\{x, y\} \cap E=\{x, y\} \\ 4 & \text { if }\{x, y\} \cap E \neq\{x, y\}\end{cases}
$$

by (II.1), $J$ is a generalized quasi-pseudodistance on $X$. Let $T: X \rightarrow X$ be a map given by the formula

$$
T(x)= \begin{cases}0 & \text { if } x \in\{0\} \cup(5,6), \\ 6 & \text { if } x \in(0,1] \cup(2,3) \cup\{6\}, \\ x / 2+3 / 2 & \text { if } x \in(1,2], \\ 3 & \text { if } x \in[3,5] .\end{cases}
$$

(III.1) $(X, p)$ is a left sequentially complete quasi-pseudometric space. Indeed, if $\left(w_{m}: m \in \mathbb{N}\right)$ is a left Cauchy sequence on $X$, then there exists $6 \in X$ such that $\forall_{\varepsilon>0} \exists_{k \in \mathbb{N}} \forall_{m, n \in \mathbb{N} ; k \leq m \leq n}\left\{p\left(6, w_{m}\right)=0<\varepsilon\right\}$, i.e., $\lim _{m \rightarrow \infty}^{L} w_{m}=6$. Thus $(X, p)$ is a left sequentially complete quasi-pseudometric space.

(III.2) The map $T$ satisfies condition (S1) for $\lambda=1 / 3$. Indeed, if $x \in X$ is arbitrary and fixed, then the following four cases hold.

Case 1. Fixing $x \in\{0\} \cup(5,6)$, by (4.4), we obtain $T(x)=T^{[2]}(x)=0 \in E$. Hence, by (4.3) and (4.1), we have $J\left(T(x), T^{[2]}(x)\right)=p(0,0)=0$. This gives that condition (S1) holds.

Case 2. Fix an arbitrary $x \in(0,1] \cup(2,3) \cup\{6\}$. By $(4.4), T(x)=T^{[2]}(x)=6 \in E$. Then, we obtain from (4.3) and (4.1) that $J\left(T(x), T^{[2]}(x)\right)=p(6,6)=0$ and thus condition (S1) holds.

Case 3. Let $x \in(1,2]$ be fixed. Note that $x \notin E$ and, by (4.4), $T(x)=x / 2+3 / 2 \in(2,3) \subset E$, $T^{[2]}(x)=6 \in E, T(x)<T^{[2]}(x)$. By (4.3) and (4.1), we also have $J\left(T(x), T^{[2]}(x)\right)=p(x / 2+$ $3 / 2,6)=1$ and $J(x, T(x))=J(x, x / 2+3 / 2)=4$. This implies $J\left(T(x), T^{[2]}(x)\right)=1 \leq 4 / 3=(1 / 3)$. $4=\lambda J(x, T(x))$. Therefore, for $\lambda=1 / 3$, condition (S1) holds.

Case 4. Let $x \in[3,5]$. By (4.4), $T(x)=T^{[2]}(x)=3 \in E$. Hence, by (4.2) and (4.1), $J\left(T(x), T^{[2]}(x)\right)=J(3,3)=p(3,3)=0$. Therefore, for $\lambda=1 / 3$, condition (S1) holds.

(III.3) $T^{[2]}$ is left quasi-closed on $X$. Indeed, we have

$$
T^{[2]}(x)= \begin{cases}0 & \text { if } x \in\{0\} \cup(5,6), \\ 3 & \text { if } x \in[3,5] \\ 6 & \text { if } x \in(0,3) \cup\{6\}\end{cases}
$$

and $T^{[2]}(X)=\{0,3,6\}$. Let $\left(w_{m}: m \in \mathbb{N}\right)$ be an arbitrary and fixed sequence in $T^{[2]}(X)$, left convergent to each point of a nonempty set $W=\left\{w \in X: \lim _{m \rightarrow \infty}^{L} w_{m}=w\right\}$ and having subsequences $\left(v_{m}: m \in \mathbb{N}\right) \subset T^{[2]}(X)$ and $\left(u_{m}: m \in \mathbb{N}\right) \subset T^{[2]}(X)$ satisfying $\forall_{m \in \mathbb{N}}\left\{v_{m}=\right.$ $\left.T^{[2]}\left(u_{m}\right)\right\}$. Clearly, $W \subset V=\left\{w \in X: \lim _{m \rightarrow \infty}^{L} v_{m}=w\right\}$ and $W \subset U=\left\{w \in X: \lim _{m \rightarrow \infty}^{L} u_{m}=\right.$ $w\}$. Hence, by (4.5), $\left(v_{m}: m \in \mathbb{N}\right) \subset\{0,3,6\}$ and $\left(u_{m}: m \in \mathbb{N}\right) \subset\{0,3,6\}$, which gives the following.

Case 1. If $\left(w_{m}: m \in \mathbb{N}\right)$ and $\left(v_{m}: m \in \mathbb{N}\right)$ are such that $\exists_{m^{\prime} \in \mathbb{N}} \forall_{m \geq m^{\prime}}\left\{v_{m}=0\right\}$, then also $\forall_{m \geq m^{\prime}}\left\{u_{m}=0\right\}$. Consequently, by Definition 2.3(ii) and Example 4.1, $V=U=[0,6]$. 
Case 2. If $\left(w_{m}: m \in \mathbb{N}\right)$ and $\left(v_{m}: m \in \mathbb{N}\right)$ are such that

$$
\exists_{m^{\prime} \in \mathbb{N}} \forall_{m \geq m^{\prime}}\left\{v_{m}=3\right\}
$$

or

$$
\exists_{m^{\prime \prime} \in \mathbb{N}} \forall_{m \geq m^{\prime \prime}}\left\{v_{m} \leq 3\right\} \quad \text { and } \quad \forall_{m^{\prime \prime \prime} \in \mathbb{N}} \exists_{m_{1} \geq m^{\prime \prime \prime}} \exists_{m_{2} \geq m^{\prime \prime \prime}}\left\{v_{m_{1}}=0 \wedge v_{m_{2}}=3\right\}
$$

then also

$$
\forall_{m \geq m^{\prime}}\left\{u_{m}=3\right\}
$$

or

$$
\forall_{m \geq m^{\prime \prime}}\left\{u_{m} \leq 3\right\} \quad \text { and } \quad\left\{u_{m_{1}}=0 \wedge u_{m_{2}}=3\right\}
$$

respectively. Consequently, $V=U=[3,6]$.

Case 3. If $\left(w_{m}: m \in \mathbb{N}\right)$ and $\left(v_{m}: m \in \mathbb{N}\right)$ are such that $\forall_{m^{\prime} \in \mathbb{N}} \exists_{m_{1} \geq m^{\prime}}\left\{v_{m_{1}}=6\right\}$, then also $u_{m_{1}}=6$. Consequently, $V=U=\{6\}$.

Of course, since $\left(w_{m}: m \in \mathbb{N}\right) \subset T^{[2]}(X)=\{0,3,6\}$, therefore $6 \in W$ in Cases 1-3. Finally, we see that $\exists_{w=6 \in W}\left\{w=T^{[2]}(w)\right\}$ in Cases 1-3. By Definition 2.4, $T^{[2]}$ is left quasi-closed in $X$.

(III.4) All the assumptions and assertions of Theorem 2.1 hold. It is straightforward to verify that $\forall_{w^{0} \in X}\left\{M\left(w^{0}\right) \neq \varnothing\right\}, \forall_{w^{0} \in X} \exists_{w=6 \in M\left(w^{0}\right)}\left\{\lim _{m \rightarrow \infty}^{L} T^{[m]}\left(w^{0}\right)=w\right\}, \operatorname{Fix}\left(T^{[2]}\right)=\operatorname{Fix}(T)=$ $\{0,3,6\}$ and $J(0,0)=J(3,3)=J(6,6)=0$.

We note that the existence of a generalized quasi-pseudodistance such that $J \neq p$ is essential.

Example 4.4 Let $(X, p)$ and $T$ be such as in Example 4.3.

(IV.1) $T$ does not satisfy condition (S1) for $J=p$. In fact, if $\exists_{\lambda \in[0,1)} \forall_{x \in X}\left\{p\left(T(x), T^{[2]}(x)\right) \leq\right.$ $\lambda p(x, T(x))\}$ holds and $w^{0}=3 / 2$, then $T\left(w^{0}\right)=9 / 4, T^{[2]}\left(w^{0}\right)=6$ and, by Example 4.1 and formulae (4.1) and (4.5), $1=p(9 / 2,6)=p\left(T\left(w^{0}\right), T^{[2]}\left(w^{0}\right)\right) \leq \lambda p\left(w^{0}, T\left(w^{0}\right)\right)=\lambda p(3 / 2,9 / 4)=$ $\lambda \cdot 1<1$. This is absurd.

Next, we notice that the assumption that $T^{[q]}$ is left quasi-closed on $X$ for some $q \in \mathbb{N}$ is essential.

Example 4.5 Let $(X, p)$ be such as in Example 4.3. Let $T: X \rightarrow X$ be of the form

$$
T(x)= \begin{cases}3 & \text { if } x \in\{0\} \cup(5,6] \\ 6 & \text { if } x \in(0,3) \\ x / 2+3 / 2 & \text { if } x \in[3,5]\end{cases}
$$

(V.1) $T$ satisfies (S1) for $J=p$ and for each $\lambda \in[0,1)$. Indeed, we have the following.

Case 1. Fixing $x \in\{0\} \cup(5,6]$, by (4.6), we obtain $T(x)=3, T^{[2]}(x)=3$. Therefore, by Example 4.1, $p\left(T(x), T^{[2]}(x)\right)=0$. This implies that condition (S1) holds. 
Case 2. Fix an arbitrary $x \in(0,3)$. Then, by $(4.6), T(x)=6, T^{[2]}(x)=3$ and, by Example 4.1, $p\left(T(x), T^{[2]}(x)\right)=p(6,3)=0$. Thus (S1) holds.

Case 3. Let $x \in[3,5]$ be fixed. By (4.6), $T(x)=x / 2+3 / 2 \in[3,4]$ and $T^{[2]}(x)=x / 4+9 / 4 \in$ $[3,13 / 4] \subset[3,4)$. However, $x \geq T(x) \geq T^{[2]}(x)$. Hence, by Example 4.1, $p\left(T(x), T^{[2]}(x)\right)=0$. This also gives (S1) for all $\lambda \in[0,1)$.

(V.2) For each $q \in \mathbb{N}$, the map $T^{[q]}$ is not left quasi-closed in $X$. Indeed, if $q \in \mathbb{N}$ is arbitrary and fixed, then, fixing $w^{0} \in(3,5)$, we get by (4.6) that $\forall_{m \in \mathbb{N}}\left\{T^{[m]}\left(w^{0}\right)=w^{0} / m+3 \sum_{n=1}^{m} 2^{-n} \wedge\right.$ $\left.w^{0}>T^{[m]}\left(w^{0}\right)>T^{[m+1]}\left(w^{0}\right)\right\}$ and that a sequence $\left(w_{m}=T^{[q+m-1]}\left(w^{0}\right): m \in \mathbb{N}\right) \subset T^{[q]}(X)$ satisfies $\forall_{m \in \mathbb{N}}\left\{3<w_{m}<4\right\}$ and is left converging to each point of the set $W=(3,6] \subset X$.

Let now $\left(v_{m}: m \in \mathbb{N}\right)$ be a sequence of the form $\forall_{m \in \mathbb{N}}\left\{v_{m}=w_{m+q}\right\}$ and let $\left(u_{m}: m \in \mathbb{N}\right)$ be a sequence of the form $\forall_{m \in \mathbb{N}}\left\{u_{m}=w_{m}\right\}$; of course, $W=V=U$, where $V=\{w \in X$ : $\left.\lim _{m \rightarrow \infty}^{L} v_{m}=w\right\}$ and $U=\left\{w \in X: \lim _{m \rightarrow \infty}^{L} u_{m}=w\right\}$. Then $\forall_{m \in \mathbb{N}}\left\{v_{m}=T^{[q]}\left(u_{m}\right)\right\}$.

Now we see that $\forall_{w \in W}\left\{w \neq T^{[q]}(w)\right\}$. This means that the map $T^{[q]}$ is not left quasi-closed in $X$.

(V.3) In summary:

(a) $(X, p)$ is a left sequentially complete quasi-pseudometric space (see (III.1)).

(b) $T$ satisfies (S1) for $J=p$ and for each $\lambda \in[0,1)$.

(c) We calculate that

$$
M\left(w^{0}\right)= \begin{cases}{[3,6]} & \text { if } w^{0} \in[0,3] \cup[5,6] \\ (3,6] & \text { if } w^{0} \in(3,5)\end{cases}
$$

and thus, for $J=p$, Theorem 2.1(A) holds.

(d) For each $q \in \mathbb{N}$, the map $T^{[q]}$ is not a left quasi-closed in $X$ and thus the assumption $\left(b_{1}\right)$ in Theorem 2.1(B) does not hold. Since $3 \in \operatorname{Fix}(T)$ thus assertion (B1) holds. Fixing $w^{0} \in(3,5)$, by $(4.7)$, we get that the sequence $\left(w^{m}=T^{[m]}\left(w^{0}\right): m \in \mathbb{N}\right)$ is not left convergent to 3 and $3 \notin M\left(w^{0}\right)=(3,6]$ and thus, for $J=p$, the assertion (B2) of Theorem 2.1 does not hold.

(e) $(X, p)$ is Hausdorff (see (I.2)), $3 \in \operatorname{Fix}(T) \neq \emptyset$ and $p(3,3)=0$. This means that, for $J=p$, Theorem 2.1(C) holds.

We compare Theorem 2.1 and [32].

Example 4.6 Let $(X, p)$ and $T$ be such as in Example 4.3.

(VI.1) $T$ is not a generalized contraction of Reilly type [32]. Indeed, suppose that $\exists_{\lambda_{0} \in[0,1)} \forall_{x, y \in X}\left\{p(T(x), T(y)) \leq \lambda_{0} p(x, y)\right\}$. Obviously, this inequality holds for $x_{0}=3 / 2$ and $y_{0}=9 / 4$ and since, by (4.4), $T\left(x_{0}\right)=9 / 4$ and $T\left(y_{0}\right)=6$, thus, by $(4.1)$, we get $1=p(9 / 4,6)=$ $p\left(T\left(x_{0}\right), T\left(y_{0}\right)\right) \leq \lambda_{0} p\left(x_{0}, y_{0}\right)=\lambda_{0} p(3 / 2,9 / 4)=\lambda_{0} \cdot 1<1$. This is absurd.

At the end of this paper, in Examples 4.7 and 4.8, we illustrate Theorem 2.1 when $(X, p)$ is not Hausdorff.

Example 4.7 Let $X=[0,1]$, let $A=\left\{1 / 2^{n}: n \in \mathbb{N}\right\}$ and let $p: X \times X \rightarrow[0, \infty)$ be of the form

$$
p(x, y)=\left\{\begin{array}{ll}
0 & \text { if } x=y \text { or }\{x, y\} \cap A=\{x, y\}, \\
1 & \text { if } x \neq y \text { and }\{x, y\} \cap A \neq\{x, y\},
\end{array} \quad x, y \in X .\right.
$$


(VII.1) The map $p$ is quasi-pseudometric on $X$. Indeed, from (4.8), we have that $p(x, x)=0$ for each $x \in X$ and thus condition (2.1) holds.

Now, it is worth noticing that condition (2.2) does not hold only if there exists $x_{0}, y_{0}, z_{0} \in$ $X$ such that $p\left(x_{0}, z_{0}\right)>p\left(x_{0}, y_{0}\right)+p\left(y_{0}, z_{0}\right)$. This inequality is equivalent to $1>0=p\left(x_{0}, y_{0}\right)+$ $p\left(y_{0}, z_{0}\right)$, where

$$
\begin{aligned}
& p\left(x_{0}, z_{0}\right)=1, \\
& p\left(x_{0}, y_{0}\right)=0
\end{aligned}
$$

and

$$
p\left(y_{0}, z_{0}\right)=0 \text {. }
$$

Conditions (4.10) and (4.11) imply $x_{0}=y_{0}$ or $\left\{x_{0}, y_{0}\right\} \subset A$ and $y_{0}=z_{0}$ or $\left\{y_{0}, z_{0}\right\} \subset A$, respectively. We consider the following four cases:

Case 1. If $x_{0}=y_{0}$ and $y_{0}=z_{0}$, then $x_{0}=z_{0}$ which, by (4.8), implies $p\left(x_{0}, z_{0}\right)=0$. By (4.9) this is absurd.

Case 2. If $x_{0}=y_{0}$ and $\left\{y_{0}, z_{0}\right\} \subset A$, then $\left\{x_{0}, z_{0}\right\} \cap A=\left\{x_{0}, z_{0}\right\}$. Hence, by (4.8), $p\left(x_{0}\right.$, $\left.z_{0}\right)=0$. By (4.9) this is absurd.

Case 3. If $\left\{x_{0}, y_{0}\right\} \subset A$ and $y_{0}=z_{0}$, then $\left\{x_{0}, z_{0}\right\} \cap A=\left\{x_{0}, z_{0}\right\}$. Hence, by (4.8), $p\left(x_{0}\right.$, $\left.z_{0}\right)=0$. By (4.9) this is absurd.

Case 4. If $\left\{x_{0}, y_{0}\right\} \subset A$ and $\left\{y_{0}, z_{0}\right\} \subset A$, then $\left\{x_{0}, z_{0}\right\} \cap A=\left\{x_{0}, z_{0}\right\}$. Hence, by (4.8), $p\left(x_{0}, z_{0}\right)=0$. By (4.9) this is absurd.

Thus, condition (2.2) holds.

We proved that $p$ is quasi-pseudometric on $X$ and $(X, p)$ is the quasi-pseudometric space.

(VII.2) $(X, p)$ is not Hausdorff. Indeed, for $x=1 / 16$ and $y=1 / 4$, we have $x \neq y$ and $\{x, y\} \cap$ $A=\{x, y\}$. Hence, by (4.8), we obtain $p(x, y)=p(y, x)=0$. This, by Definition 2.1(ii), means that $(X, p)$ is not Hausdorff.

Example 4.8 Let $X=[0,1] \subset \mathbb{R}$, let $p$ be the same as in Example 4.7 and let $T: X \rightarrow X$ be given by the formula

$$
T(x)= \begin{cases}1 / 2 & \text { if } x \in[0,1 / 4] \\ 1 / 4 & \text { if } x \in(1 / 4,1]\end{cases}
$$

(VIII.1) The space $(X, p)$ is a not Hausdorff space. See (VII.2).

(VIII.2) The space $(X, p)$ is a left sequentially complete. Indeed, let $\left(u_{m}: m \in \mathbb{N}\right)$ be a left Cauchy sequence in $X$. By (4.8), not losing generality, we may assume that

$$
\forall_{0<\varepsilon<1} \exists_{k_{0} \in \mathbb{N}} \forall_{m, n \in \mathbb{N} ; k_{0}<m<n}\left\{p\left(u_{m}, u_{n}\right)=0<\varepsilon<1\right\} .
$$

Now, we have the following two cases:

Case 1. Let $\forall_{m \in \mathbb{N} ; k_{0}<m}\left\{u_{m} \in A\right\}$. By (4.8), in particular, since $1 / 2 \in A$, we have that $\forall_{m>k_{0}}\left\{p\left(1 / 2, u_{m}\right)=0\right\}$. This, by Definition 2.3(iii), means that $\left(u_{m}: m \in \mathbb{N}\right)$ is left convergent in $X$ (we have that $\lim _{m \rightarrow \infty}^{L} u_{m}=1 / 2$ ); 
Case 2. Let $\exists_{m_{0} \in \mathbb{N} ; k_{0}<m_{0}}\left\{u_{m_{0}} \notin A\right\}$. Then we have the following two subcases:

Subcase 2(a) Let $\forall_{m \in \mathbb{N} ; k_{0}<m, m \neq m_{0}}\left\{u_{m}=u_{m_{0}}\right\}$. Then, by (4.8), we get $\forall_{m \in \mathbb{N} ; m_{0}<m}\left\{p\left(u_{m_{0}}\right.\right.$, $\left.\left.u_{m}\right)=0\right\}$ and this implies $\lim _{m \rightarrow \infty}^{L} u_{m}=u_{m_{0}}$;

Subcase 2(b) Let $\exists_{m_{1} \in \mathbb{N} ; k_{0}<m_{1}, m_{1} \neq m_{0}}\left\{u_{m_{1}} \neq u_{m_{0}}\right\}$. Then, by (4.8), since $u_{m_{0}} \notin A$ and $u_{m_{1}} \neq$ $u_{m_{0}}, p\left(u_{m_{1}}, u_{m_{0}}\right)=p\left(u_{m_{0}}, u_{m_{1}}\right)=1$. However, since $k_{0}<m_{0}$ and $k_{0}<m_{1}$, this, by (4.13), implies $p\left(u_{m_{1}}, u_{m_{0}}\right)=0$ when $m_{1}<m_{0}$ and $p\left(u_{m_{0}}, u_{m_{1}}\right)=0$ when $m_{0}<m_{1}$. This is absurd.

We proved that if (4.13) holds, then $\left\{u: \lim _{m \rightarrow \infty}^{L} u_{m}=u\right\} \neq \varnothing$. By Definition 2.3(ii), the sequence $\left(u_{m}: m \in \mathbb{N}\right)$ is left convergent in $X$.

(VIII.3) For $J=p$ the assumption (S1) of Theorem 2.1 holds (more precisely, the map $T$ satisfies condition (S1) for $J=p$ and for each $\lambda \in[0,1)$ ). This follows from the fact that, by (4.8), $p(T(x), T(y))=0$ for each $x, y \in X$.

(VIII.4) The map $T$ is not left quasi-closed on $X$. Indeed, let a sequence $\left(w_{m}: m \in \mathbb{N}\right)$ in $T(X)=\{1 / 4,1 / 2\}$ be of the form

$$
w_{m}= \begin{cases}1 / 4 & \text { if } m \text { is even } \\ 1 / 2 & \text { if } m \text { is odd }\end{cases}
$$

Since $\forall_{m \in \mathbb{N}}\left\{w_{m} \in A\right\}$ thus, by (4.8), $\forall_{w \in A}\left\{p\left(w, w_{m}\right)=0\right\}$ and $\forall_{w \in X \backslash A}\left\{p\left(w, w_{m}\right)=1\right\}$. Hence $\left\{w: \lim _{m \rightarrow \infty}^{L} w_{m}=w\right\}=A$. Moreover, its subsequences $\left(u_{m}=1 / 4: m \in \mathbb{N}\right)$ and $\left(v_{m}=1 / 2\right.$ : $m \in \mathbb{N})$ satisfy $\forall_{m \in \mathbb{N}}\left\{v_{m}=T\left(u_{m}\right)\right\}$. Clearly, $\left\{w: \lim _{m \rightarrow \infty}^{L} w_{m}=w\right\}=\left\{v: \lim _{m \rightarrow \infty}^{L} v_{m}=v\right\}=$ $\left\{u: \lim _{m \rightarrow \infty}^{L} u_{m}=u\right\}=A$. However, there does not exist $w \in A$ such that $w=T(w)$.

(VIII.5) The map $T^{[2]}$ is left quasi-closed on $X$. Indeed, we have

$$
T^{[2]}(x)= \begin{cases}1 / 4 & \text { if } x \in[0,1 / 4] \\ 1 / 2 & \text { if } x \in(1 / 4,1]\end{cases}
$$

and let $\left(w_{m}: m \in \mathbb{N}\right)$ be an arbitrary and fixed sequence in $T^{[2]}(X)=\{1 / 4,1 / 2\}$, left convergent to each point of a nonempty set $\left\{w: \lim _{m \rightarrow \infty}^{L} w_{m}=w\right\} \subset X$ and having subsequences $\left(v_{m}: m \in \mathbb{N}\right)$ and $\left(u_{m}: m \in \mathbb{N}\right)$ satisfying $\forall_{m \in \mathbb{N}}\left\{v_{m}=T^{[2]}\left(u_{m}\right)\right\}$. Thus, $\left(w_{m}: m \in \mathbb{N}\right) \subset$ $\{1 / 4,1 / 2\} \subset A,\left(v_{m}: m \in \mathbb{N}\right) \subset\{1 / 4,1 / 2\} \subset A$ and $\left(u_{m}: m \in \mathbb{N}\right) \subset\{1 / 4,1 / 2\} \subset A$. Hence, by (4.8), we conclude that

$$
\lim _{m \rightarrow \infty} p\left(w, w_{m}\right)=\lim _{m \rightarrow \infty} p\left(w, v_{m}\right)=\lim _{m \rightarrow \infty} p\left(w, u_{m}\right)= \begin{cases}0 & \text { if } w \in A, \\ 1 & \text { if } w \in X \backslash A .\end{cases}
$$

This gives $\left\{w: \lim _{m \rightarrow \infty}^{L} w_{m}=w\right\}=\left\{v: \lim _{m \rightarrow \infty}^{L} v_{m}=v\right\}=\left\{u: \lim _{m \rightarrow \infty}^{L} u_{m}=u\right\}=A$. Next, we see that $\exists_{w \in\{1 / 4,1 / 2\} \subset A=\left\{w: \lim _{m \rightarrow \infty}^{L} w_{m}=w\right\}}\left\{w=T^{[2]}(w)\right\}$. By Definition 2.4, $T^{[2]}$ is left quasiclosed on $X$.

(VIII.6) For $J=p$, the statements (A) and (B) of Theorem 2.1 hold. This follows from (VIII.1)-(VIII.5). From the above, it follows:

$$
\begin{aligned}
& \operatorname{Fix}\left(T^{[2]}\right)=\{1 / 4,1 / 2\} ; \\
& \forall_{w^{0} \in[0,1 / 4]}\left\{\left\{w: \lim _{m \rightarrow \infty}{ }^{L} T^{[m]}\left(w^{0}\right)=w\right\}=[1 / 4,1]\right\} ; \\
& \forall_{w^{0} \in(1 / 4,1]}\left\{\left\{w: \lim _{m \rightarrow \infty}{ }^{L} T^{[m]}\left(w^{0}\right)=w\right\}=[1 / 2,1]\right\} ;
\end{aligned}
$$




$$
\begin{aligned}
& \forall_{w^{0} \in[0,1 / 4]} \exists_{w=1 / 4 \in \operatorname{Fix}\left(T^{[2]}\right)}\left\{\lim _{m \rightarrow \infty}{ }^{L} T^{[m]}\left(w^{0}\right)=1 / 4\right\} ; \\
& \forall_{w^{0} \in(1 / 4,1]} \exists_{w=1 / 2 \in \operatorname{Fix}\left(T^{[2]}\right)}\left\{\lim _{m \rightarrow \infty}{ }^{L} T^{[m]}\left(w^{0}\right)=1 / 2\right\} ;
\end{aligned}
$$

and

$$
\forall_{w^{0} \in[0,1]}\left\{\operatorname{Fix}\left(T^{[2]}\right) \neq\left\{w: \lim _{m \rightarrow \infty}{ }^{L} T^{[m]}\left(w^{0}\right)=w\right\}\right\}
$$

Moreover, by (4.8), since $\operatorname{Fix}\left(T^{[2]}\right)=\{1 / 4,1 / 2\} \subset A$, thus, by (4.12), we get $p(1 / 4,1 / 2)=$ $p(1 / 2,1 / 4)=0$, so (B3) holds.

(IX.7) For $J=p$, the statement (C) of Theorem 2.1 does not hold. We have: the assumption $\left(\mathrm{c}_{1}\right)$ does not hold; for $q=2$ the assumption $\left(\mathrm{c}_{2}\right)$ holds; Fix $\left(T^{[2]}\right) \neq \varnothing$; properties $(\mathrm{C} 1)$ and (C2) do not hold since $\operatorname{Fix}(T)=\varnothing$.

Remark 4.1 (a) We see that in Example 4.3: (i) The map $T$ is not left quasi-closed in $X$ and $T^{[2]}$ is left quasi-closed in $X$; (ii) The map $T$ satisfies condition (S1) for $J$ defined by (4.3) and for $\lambda=1 / 3$; (iii) When $J=p$ then, for each $\lambda \in[0,1$ ), the map $T$ does not satisfy condition (S1) (see Example 4.4); (iv) Assumptions of Theorem 2.1 are satisfied; (v) In complete metric spaces, the assumptions of Banach [1], Rus [3], Subrahmanyam [4], Kada et al. [8, Corollary 2] and Suzuki [10, Theorem 1] theorems are not satisfied.

(b) In metric spaces $(X, p)$, the generalized pseudodistances $J$ (see Definition 2.5) generalize: metrics $p$, distances of Tataru [11], $w$-distances of Kada et al. [8], $\tau$-distances of Suzuki [23] and $\tau$-functions of Lin, Du [9]; for details, see [15, 16].

(c) It is important to observe that we provide the conditions guaranteeing the existence of fixed points and periodic points of the maps $T: X \rightarrow X$, and in our studies we determine the optimal global minima of the maps $x \rightarrow J\left(x, T^{[q]}(x)\right), q \in \mathbb{N}$.

(d) It is worth noticing that in the literature the fixed and periodic points of contractions in not Hausdorff spaces were not studied.

Competing interests

The authors declare that they have no competing interests.

Authors' contributions

All authors contributed equally. All authors read and approved the final manuscript.

Received: 28 January 2013 Accepted: 29 April 2013 Published: 15 May 2013

References

1. Banach, S: Sur les opérations dans les ensembles abstraits et leurs applications aux équations intégrales. Fundam. Math. 3, 133-181 (1922)

2. Caccioppoli, R: Un teorema generale sull'esistenza di elementi uniti in una transformazione funzionale. Rend. Accad. Naz. Lincei 11, 794-799 (1930)

3. Rus, IA: The method of successive approximations. Rev. Roum. Math. Pures Appl. 17, 1433-1437 (1972)

4. Subrahmanyam, PV: Remarks on some fixed point theorems related to Banach's contraction principle. J. Math. Phys. Sci. 8, 445-458 (1974) Erratum 9, 195 (1975)

5. Kasahara, S: On some generalizations of the Banach contraction theorem. Publ. Res. Inst. Math. Sci. 12, 427-437 $(1976 / 77)$

6. Hicks, TL, Rhoades, BE: A Banach type fixed-point theorem. Math. Jpn. 24, 327-330 (1979/80)

7. Cobzas, S: Functional Analysis in Asymmetric Normed Spaces. Birkhäuser, Basel (2013)

8. Kada, O, Suzuki, T, Takahashi, W: Nonconvex minimization theorems and fixed point theorems in complete metric spaces. Math. Jpn. 44, 381-391 (1996)

9. Lin, L-J, Du, W-S: Ekeland's variational principle, minimax theorems and existence of nonconvex equilibria in complete metric spaces. J. Math. Anal. Appl. 323, 360-370 (2006) 
10. Suzuki, T: Generalized distance and existence theorems in complete metric spaces. J. Math. Anal. Appl. 253, 440-458 (2001)

11. Tataru, D: Viscosity solutions of Hamilton-Jacobi equations with unbounded nonlinear terms. J. Math. Anal. Appl. 163 345-392 (1992)

12. Vályi, I: A general maximality principle and a fixed point theorem in uniform spaces. Period. Math. Hung. 16, 127-134 (1985)

13. Włodarczyk, K, Plebaniak, R: A fixed point theorem of Subrahmanyam type in uniform spaces with generalized pseudodistances. Appl. Math. Lett. 24, 325-328 (2011)

14. Włodarczyk, K, Plebaniak, R: Quasigauge spaces with generalized quasipseudodistances and periodic points of dissipative set-valued dynamic systems. Fixed Point Theory Appl. 2011, Article ID 712706 (2011) doi:10.1155/2011/712706

15. Włodarczyk, K, Plebaniak, R: Maximality principle and general results of Ekeland and Caristi types without lower semicontinuity assumptions in cone uniform spaces with generalized pseudodistances. Fixed Point Theory Appl. 2010, Article ID 175453 (2010)

16. Włodarczyk, K, Plebaniak, R: Periodic point, endpoint, and convergence theorems for dissipative set-valued dynamic systems with generalized pseudodistances in cone uniform and uniform spaces. Fixed Point Theory Appl. 2010 Article ID 864536 (2010)

17. Włodarczyk, K, Plebaniak, R: Kannan-type contractions and fixed points in uniform spaces. Fixed Point Theory Appl. 2011, 90 (2011). doi:10.1186/1687-1812-2011-90

18. Włodarczyk, K, Plebaniak, R: Generalized uniform spaces, uniformly locally contractive set-valued dynamic systems and fixed points. Fixed Point Theory Appl. 2012, 104 (2012). doi:10.1186/1687-1812-2012-104

19. Włodarczyk, K, Plebaniak, R: Fixed points and endpoints of contractive set-valued maps in cone uniform spaces with generalized pseudodistances. Fixed Point Theory Appl. 2012, 176 (2012). doi:10.1186/1687-1812-2012-176

20. Włodarczyk, K, Plebaniak, R: Leader type contractions, periodic and fixed points and new completivity in quasi-gauge spaces with generalized quasi-pseudodistances. Topol. Appl. 159, 3502-3512 (2012). doi:10.1016/j.topol.2012.08.013

21. Włodarczyk, K, Plebaniak, R: Contractivity of Leader type and fixed points in uniform spaces with generalized pseudodistances. J. Math. Anal. Appl. 387, 533-541 (2012). doi:10.1016/j.jmaa.2011.09.006

22. Włodarczyk, K, Plebaniak, R: Contractions of Banach, Tarafdar, Meir-Keller, Ćirić-Jachymski-Matkowski and Suzuki types and fixed points in uniform spaces with generalized pseudodistances. J. Math. Anal. Appl. (2013). doi:10.1016/j.jmaa.2013.03.030

23. Suzuki, T: Subrahmanyam's fixed point theorem. Nonlinear Anal. 71, 1678-1683 (2009)

24. Suzuki, T: Convergence of the sequence of successive approximations to a fixed point. Fixed Point Theory Appl. 2010 Article ID 716971 (2010). doi:10.1155/2010/716971

25. Suzuki, T: Several fixed point theorems concerning $\tau$-distances. Fixed Point Theory Appl. 2004(3), 195-209 (2004)

26. Kikkawa, M, Suzuki, T: Some similarity between contractions and Kannan mappings. Fixed Point Theory Appl. 2008, Article ID 649749 (2008). doi:10.1153/2008/649749

27. Reilly, IL, Subrahmanyam, PV, Vamanamurthy, MK: Cauchy sequences in quasi-pseudo-metric spaces. Monatshefte Math. 93, 127-140 (1982)

28. Reilly, IL: Quasi-gauge spaces. J. Lond. Math. Soc. 6, 481-487 (1973)

29. Wilson, WA: On quasi-metric-spaces. Am. J. Math. 53, 675-684 (1931)

30. Albert, GA: A note on quasi-metric spaces. Bull. Am. Math. Soc. 47, 479-482 (1941)

31. Kelly, JC: Bitopological spaces. Proc. Lond. Math. Soc. 13, 71-89 (1963)

32. Reilly, IL: A generalized contraction principle. Bull. Aust. Math. Soc. 10, 349-363 (1974)

doi:10.1186/1687-1812-2013-128

Cite this article as: Włodarczyk and Plebaniak: Asymmetric structures, discontinuous contractions and iterative approximation of fixed and periodic points. Fixed Point Theory and Applications 2013 2013:128.

\section{Submit your manuscript to a SpringerOpen ${ }^{\circ}$ journal and benefit from:}

- Convenient online submission

- Rigorous peer review

- Immediate publication on acceptance

- Open access: articles freely available online

- High visibility within the field

- Retaining the copyright to your article 